\title{
The relationship between HMGB1 and immune infiltration serves as a treatment target molecule in different cancer tissues: May be used therapeutic target for possible SARS- CoV-2 infection
}

\author{
(1) Rahşan Ilıkçı Sağkan¹, (D) Dilara Fatma Akın-Balı² \\ 1 Usak University Faculty of Medicine, Department of Medical Biology, Usak, Turkey \\ 2Nigde Omer Halisdemir University Faculty of Medicine, Department of Medical Biology, Nigde, Turkey
}

Date submitted:

31.12.2020

Date accepted:

25.01.2021

\section{Online publication date:}

15.12.2021

\section{Corresponding Author:}

Rahşan Ilıkçı Sağkan, M.D., Usak

University Faculty of Medicine,

Department of Medical Biology, Usak,

Turkey

rahsan.likci@usak.edu.tr

ORCID:

orcid.org/0000-0003-3844-6158

Keywords: HMGB1, COVID-19, SARS-CoV-2, therapy, immune infiltration

Presented in: $25^{\text {th }}$ National

Immunology Congress 20-22

November 2020 Digital Congress.

\begin{abstract}
Aims: To illustrate which tumor cells may express high-mobility group box 1 (HMGB1), which is also Coronavirus disease-2019 (COVID-19) target molecule as a treatment option for possible severe acute respiratory syndrome-Coronavirus-2 (SARS-CoV-2) infection.

Methods: We investigated m-RNA expression patterns of HMGB1 in 33 different cancer tissues. HMGB1 m-RNA expression profiles were compared with the Gene Expression Profiling Interactive Analysis database. Comparisons of promoter methylation levels with the UALCAN database were also performed. Finally, the correlation between HMGB1 and immune cells was investigated by using TIMER tool.

Results: High expression profile of HMGB1 was determined in 8 different cancer tissues (colon adenocarcinoma, diffuse large B-cell lymphoma, glioblastoma multiforme, brain lower grade glioma, pancreatic adenocarcinoma, rectum adenocarcinoma, stomach adenocarcinoma and thymoma) when compared with the healthy tissues $(p<0.05)$. The promoter methylation level of HGMB1 in different cancers was significantly lower. In addition, the level of expression and overall survival did not correlate in studied tumor samples. HMGB1 transcription level was associated with innate (monocyte, neutrophil) and adaptive immune cells (cytotoxic $T$ lymphocyte and B cell) in tumor samples.
\end{abstract}

Conclusions: The use of agents that inhibit HMGB1 protein may offer an effective approach, not only against the cancer cell proliferation, but also as a strategy to minimize the possible SARS-CoV-2 infection in cancer patients with high HMGB1 expression.

\section{Introduction}

Cancer is a term that collectively describes a group of apparently unrelated conditions that have common immune response related pathways leading to various tissue damage (1). Although each of these cancer types has distinctive molecular background and pathophysiology, the dysregulated immune response is believed to be pivotal to diseases' pathogenesis. High-mobility group box 1 (HMGB1), a member of the HMG protein family, is one of the damage-associated molecular patterns (DAMPs) which are danger molecules released from damaged cells and activate the innate immune system components (2-4). Innate immune system is the first defense mechanism against the pathogenic microorganisms such as viruses, bacteria, and parasites. DAMPs have a dual role by contributing host's defense and promoting pathological inflammatory responses $(5,6)$. Experimental studies have demonstrated that inflammatory 
signaling pathway was induced and inflammatory cytokines including interleukin 6 (IL-6), interleukin 1 (IL-1), tumor necrosis factor (TNF) were produced by releasing of HMGB1 molecule to the extracellular region $(6,7)$. HMGB1 is a crucial molecular target for infectious diseases, ischemia, immune diseases, neurodegenerative diseases, metabolic diseases and cancer $(2,4,8)$. HMGB1 has many biological activities in normal and cancerous cells and regulates many basic molecular events such as transcription, replication, recombination, DNA repair, genomic stabilization and TLR4 activation $(5,8,9)$. In the coordination of the cell's stress response, not only in intracellular functions such as chromosome protector, autophagy, and apoptotic cell death inhibitor, but also outside the cell, it plays a critical role as the DAMP prototype $(6,9)$. The interplay of DAMP and other factors releases cytokine, chemokine, and growth factor activity and manages inflammation and immune response. The global public health is novel coronavirus [Severe acute respiratory syndrome-Coronavirus-2 (SARS-CoV-2) - also known as 2019-nCoV], the RNA virus that causes Coronavirus disease-2019 (COVID-19) $(10,11)$. It is important to understand the role of HMGB1 and associated cellular mechanism including transcription, methylation, immune response, prognosis in hypothetically infection with SARS-CoV-2 in various tumor tissues and healthy tissue. In humans, SARS-CoV-2 infection results in a diversity of clinical manifestations. While several genetic and epigenetic factors associated with COVID-19 have been identified, pathogenesis of COVID-19 in cancer patients is still poorly understood. There is strong evidence implicating the involvement of the immune reaction in the progression to COVID-19, tumor infiltrating immune cells playing a role $(12,13)$. For this reason, higher HMGB1 expression in cancer patients increases the risk for developing infectious diseases. In this study, we mainly discuss our own data on the analysis of the intratumoral infiltrates in human cancers, their relationship with HMGB1 expression, the respective importance in view of their prognostic value and interaction with possible SARS-CoV-2 infection. To illustrate the general characteristic of the host HMGB1 expression-SARS-CoV-2 interactions, eight examples of the human tumors will be analyzed by using bioinformatics tools. Finally, we will propose some new insights on which tumor cells may express HMGB1, which is also COVID-19 target molecule to treatment and protection from possible SARS-CoV-2 infection.

\section{Methods}

\section{Subjects}

This study was carried out by using The Cancer Genome Atlas (TCGA) data sets of 33 different cancer types.

\section{Expression and Correlation Analysis}

Gene Expression Profiling Interactive Analysis (GEPIA) is a web server that allows the evaluation of RNA expression of normal tissue samples as a control group and tumor tissue samples on a wide scale obtained within the scope of TCGA and GTEx projects. To identify HMGB1 gene expression profile, we compared box plot from 33 different cancer tissue samples and healthy tissue samples to GEPIA database (14). In addition, overall survival analysis was performed based on the Log-rank test with a $95 \%$ confidence interval in order to create survival graphs. The $p$ values were calculated automatically by the tool and $p$ values under 0.05 were considered significant. HMGB1 expression profile screening was performed in 33 different cancer tissues [adrenal cancer, bladder and urothelial cancers, breast cancer, cervix cancer, colorectal cancer, lymphoma, esophageal cancer, glioblastoma, head and neck cancer, renal cancer, leukemia, liver cancer, lung cancer, ovarian cancer, stomach cancer, pancreatic cancer, prostate cancer, brain tumor, uterine cancer, mesothelioma, melanoma, sarcoma, thyroid cancer, thymoma (THYM), uterine cancer]. A statistically significant difference was found in terms of HMGB1 gene expression in 8 tumor tissues including colon adenocarcinoma (COAD), lymphoid neoplasm diffuse large B-cell lymphoma (DLBC), glioblastoma multiforme (GBM), brain lower grade glioma (LGG), pancreatic adenocarcinoma (PAAD), rectum adenocarcinoma (READ), stomach adenocarcinoma (STAD), THYM among these cancer types. Further analyses were performed on these samples.

\section{Analysis of Promoter Methylation Levels}

UALCAN is an interactive open-access web page for the analysis of OMICS data (http://ualcan.Path.uab.edu/index.html). This database is built on PERL-CGI and can be used in about 6000 different gene methylation levels (15). So, the promoter methylation level of $H M G B 1$ gene was evaluated with this database in different cancer tissues.

\section{Comprehensive Analysis of Tumor-infiltrating Immune Cells}

TIMER is a comprehensive open access online database that can analyze immune invasion levels and differences in gene expression levels in different tumor tissues (16). Correlation between HMGB1 expression and immune infiltrating cells [B cells, CD4+ T cells, CD8+ T cells, neutrophils, macropages, and dendritic cells (DCs)] was analyzed by using the TIMER database.

\section{Statistical Analysis}

We used the GEPIA database to examine the statistical significance $(p<0.05)$ by analyzing all parameters. Five statistical methods including Kaplan-Meier curves, log-rank test, Pearson test, Spearman's correlation, and Student's t-test were used in overall survival, comparison of low and high groups, correlation analyses, evaluation of HMGB1 with immune infiltration, and comparison of two independent samples, respectively. 


\section{Results}

\section{Results of Expression and Correlation Analysis}

Initially, gene expression profile belonging to the 33 different cancer tissues was compared with healthy tissues and evaluated separately in terms of HMGB1 expression profiles using the GEPIA online tool in order to investigate changes in the gene expression level of HMGB1 in the cancer tissue (Figure 1). As a result of our analysis, it was determined that the expression profile of HMGB1 was statistically significant in 8 different cancer tissues (COAD, DLBC, GBM, LGG, PAAD, READ, STAD and THYM). Therefore, 8 different tumor tissues were included in all analyses made thereafter. In our study, HMGB1 was found to have a statistically significantly higher expression level in these 8 different tumor tissues when compared to healthy tissue samples (Figure 2, p <0.05). Then, the same datasets were used in order to analyze the overall survival of 8 different cancer patients according to HMGB1 expression (Figure 3). According to the results of survival analysis, HMGB1 high expression levels were found to be associated with good prognosis in cancer patients with THYM $(p<0.05)$. No statistically significant difference was found between low and high expression levels for other types of cancer.

\section{Analysis of the Promoter Methylation Levels}

DNA methylation is an important case in the epigenetic modification of the genome and is closely related to the development process of cancer. Hypo methylation may cause genome instability and activate the related genes. It was determined that HMGB1 expression profile had high levels of expression in 8 different cancers. According to the results of our analysis, we performed using the UALCAN online tool in order to determine the level of DNA methylation, the promoter methylation level of HMGB1 was found to be lower than healthy tissues (hypo methylation) (Figure 4). GBM and LGG were not included in the analysis as the UALCAN database did not provide access to their comparison with healthy tissues.

\section{Comprehensive Analysis of Tumor-infiltrating Immune Cells}

When we analyzed the correlation between m-RNA expression level of HMGB1 and immune infiltration profile in different cancers, the correlation was detected between HMGB1 expression and immune infiltration in COAD, DLBC, GBM, LGG, PAAD, READ, THYM cancer types according to TIMER database uploaded data. HMGB1 expression was positively correlated with B cell $(r=0.11, p<0.05), C D 8+T$ cell $(r=0.208$, $p<0.0001)$, macrophage $(r=0.112, p<0.05)$ infiltration in COAD; $B$ cell $(r=0.688, p<0.05)$ in DLBC; $B$ cell $(r=0.124, p<0.05)$, CD8+ T cell $(r=0.25, p<0.001)$, macrophage $(r=0.144, p<0.001)$, neutrophil $(r=0.136, p<0.001)$ in GBM; macrophage $(r=0.109$, $p<0.05)$ in LGG; B cell $(r=0.282, p<0.001), C D 8+T$ cell $(r=0.298$, $p<0.0001)$, macrophage $(r=0.298, \quad p<0.00001)$, neutrophil $(r=0.285, p<0.0001), D C s(r=0.351, p<0.0001)$ in PAAD; CD $8+T$

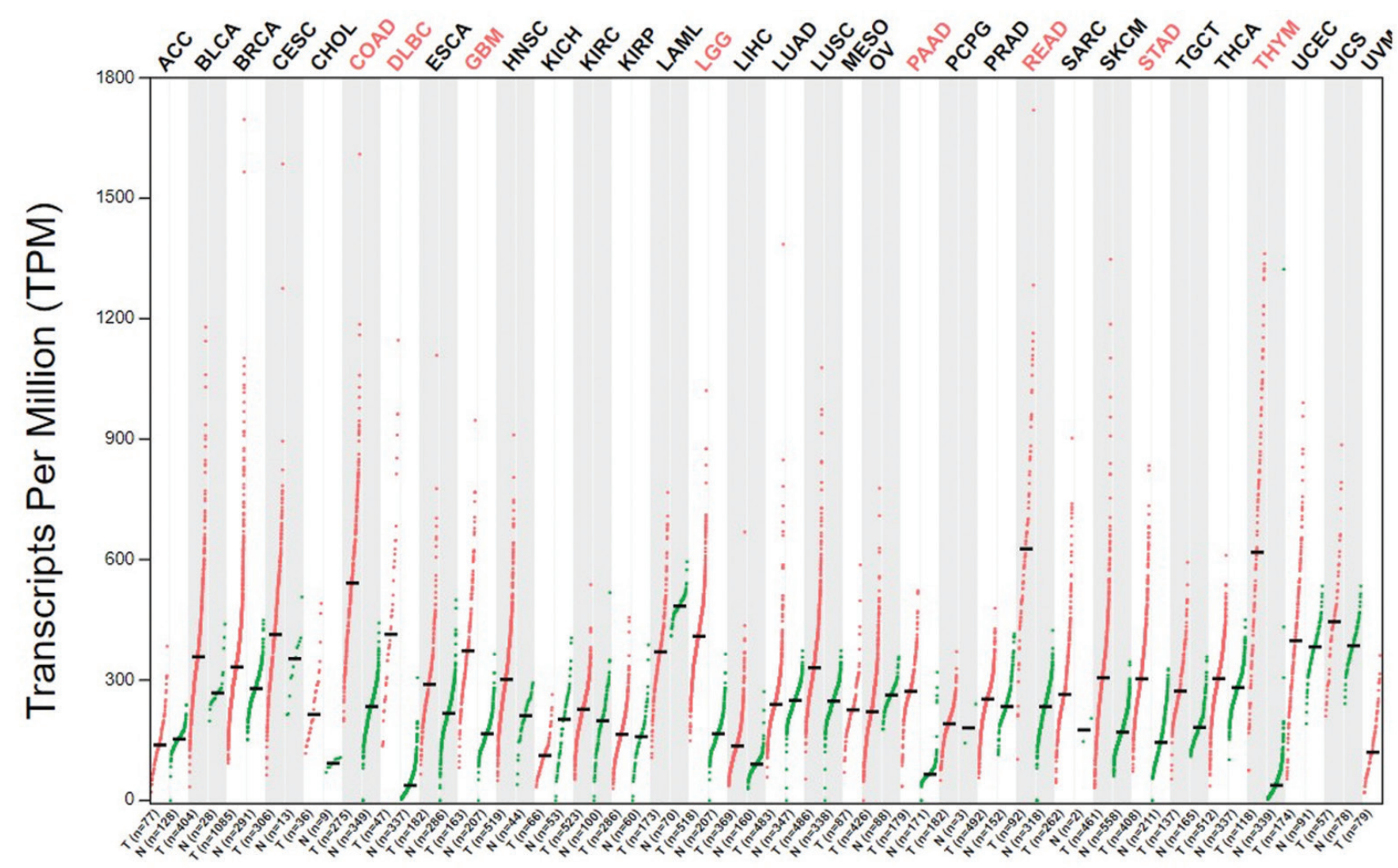

Figure 1. HMGB1 in different cancers compared to normal tissues in the Gene Expression Profiling Interactive Analysis database 

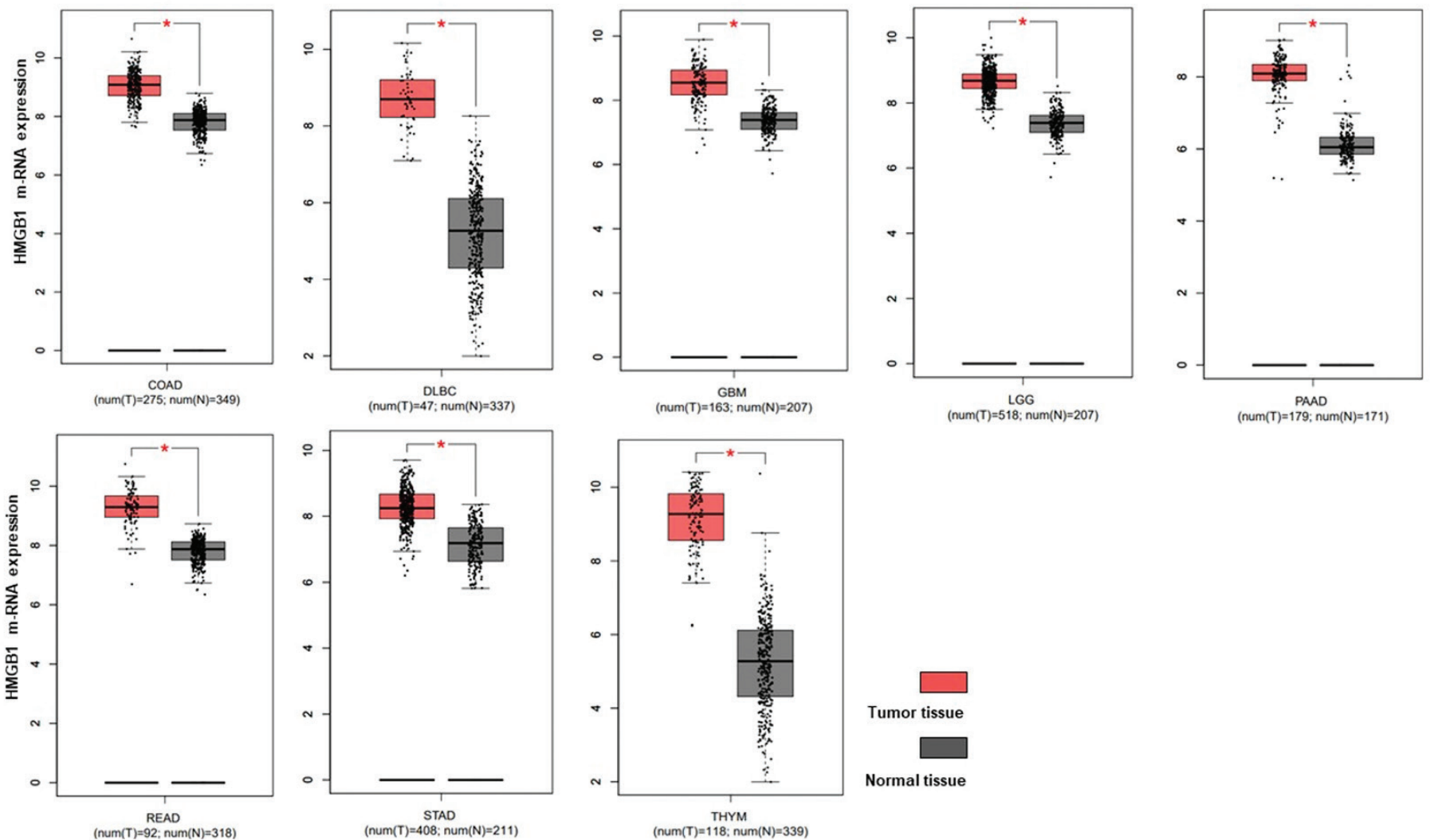

Figure 2. Comparative analysis of the tissue-specific differential expression of HMGB1 gene in different cancer tissues using GEPIA (*indicates $\mathrm{p}<0.05)$
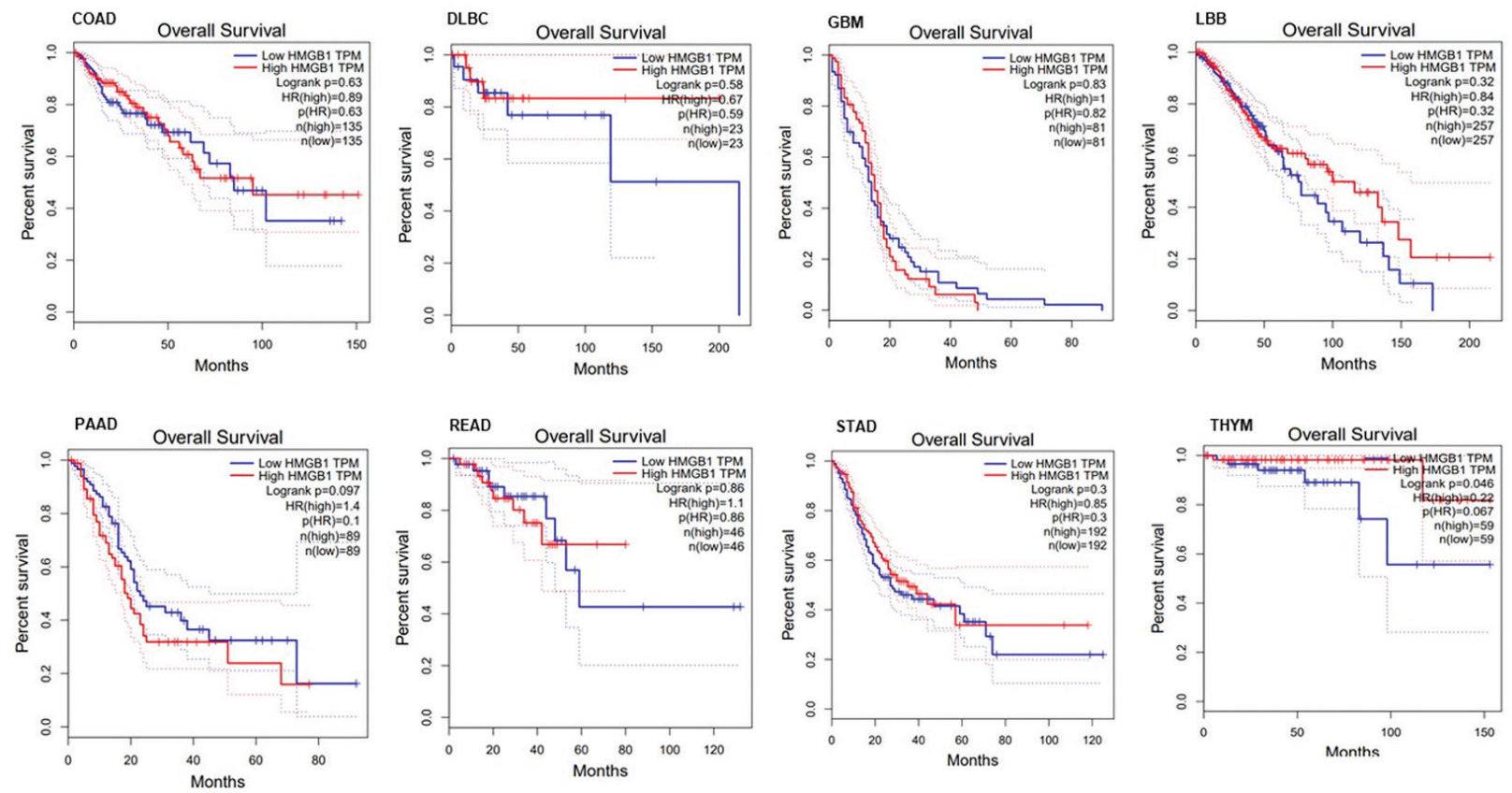

Figure 3. Comparison of Kaplan-Meier survival curves of the high and low expressions of HMGB1 in different cancer tissues

cell $(r=0.191, p<0.05)$, neutrophil $(r=0.187, p<0.05)$ in READ; $B$ cell $(r=0.71, p<0.0001), C D 8+T$ cell $(r=0.566, p<0.0001)$, CD4+ T cell $(r=0.723, p<0.0001)$, macrophage $(r=0.6, p<0.0001)$, DCs $(r=0.781, p<0.0001)$ in THYM patients' tumor samples compared to healthy samples. However, in silico analysis results showed that limited or weak correlation was detected between CD4+ T cell and HMGB1 expression level in all studied tumor tissues except THYM tumor samples (Figures $5 a, 5 b$ ). 

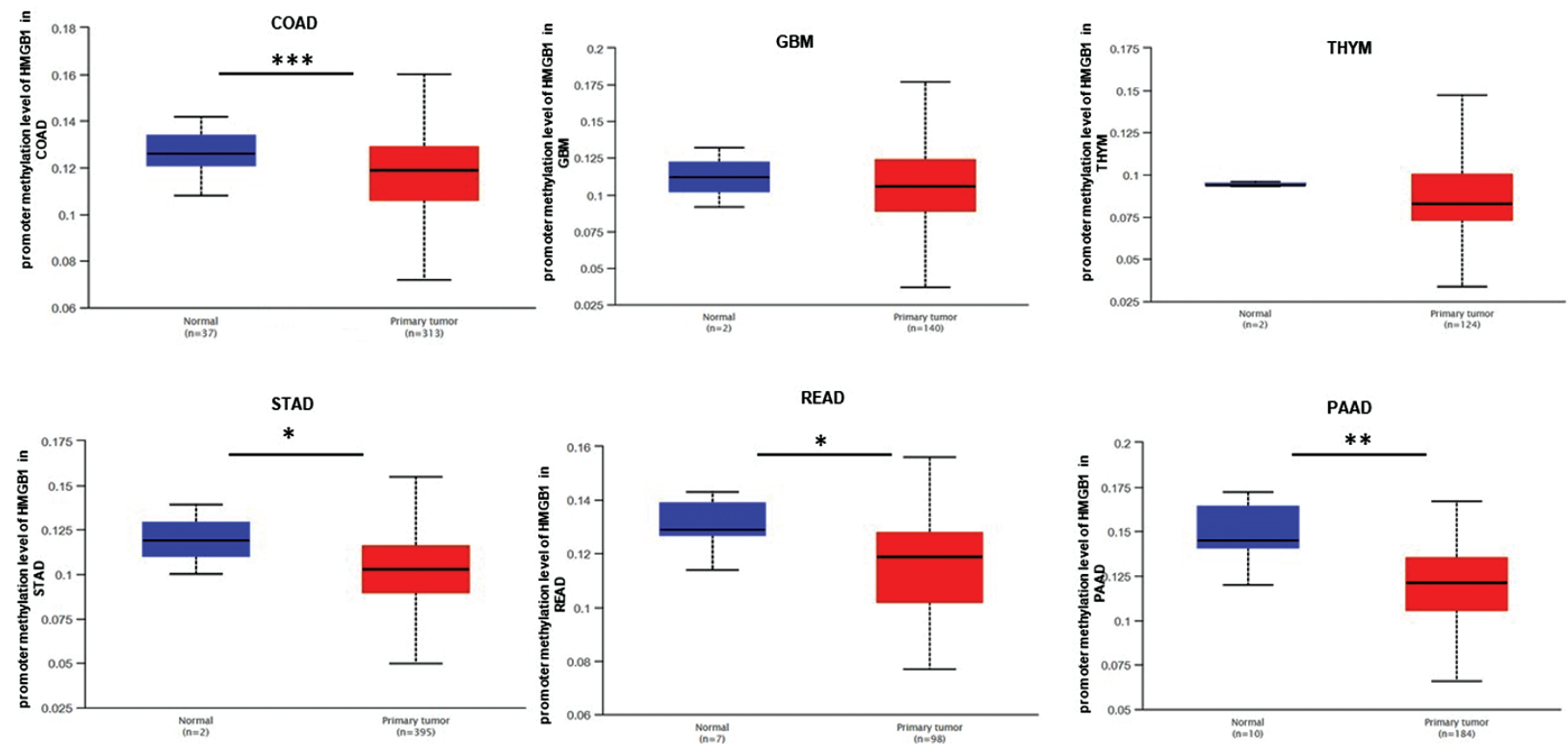

Figure 4. The promoter methylation level of HMGB1 in different cancer tissues

\section{Discussion}

It has been shown that HMGB1 may support tumor development and progression by inducing chronic inflammation and, in contrast, this molecule may inhibit tumor progression $(17,18)$. A number of studies have reported pathophysiological roles of HMGB1 in various diseases, including infectious diseases and cancer $(3,5,17,18)$. It is noteworthy that the up-regulation of HMGB1 in cancer is due to the fact that cancer cells are exposed to different stress parameters such as acidosis, hypoxia and inflammation. A number of studies reported anti-tumor immune stimulatory roles of extracellular-HMGB1 $(19,20)$. Treatment based on HMGB1 can be useful against cancers and viral infection. Extracellular-HMGB1 released from damaged cells as a danger signal can stimulate DCs, followed by the release of pro-inflammatory cytokines $(9,21)$. Moreover, some analysis results showed that expression of HMGB1 could promote the cross-presentation of peptide antigens to major histocompatibility complex class 1 in DCs, activating cytotoxic T lymphocytes (CTLs) $(2,4,9,22)$. From the aspect of this mechanism, the roles of extracellular HMGB1 activating both innate immunity and adaptive immunity may be an important molecular mechanism in response to viral infection including SARS-CoV-2 for cancer patients $(23,24)$. Andersson et al. $(23)$ reported that extracellular HMGB1 might be therapeutic target in COVID-19. Based on this information, which cancer types expressed this danger molecule and what is the relationship between HMGB1 and immune cells? Here, we evaluated this in silico analysis on the role of HMGB1 in various cancer tissues including COAD, DLBC, GBM, LGG, PAAD, READ, STAD and THYM and healthy samples and the m-RNA expression of HMGB1 was higher in eight cancer types according to the results of expression analyses. We also concluded the possibility of HMGB1 as therapeutic targets for COVID-19. In cases where cancers including COAD, DLBC, GBM, LGG, PAAD, READ, STAD and THYM are high, HMGB1 is the treatment option for these cancer patients infected with SARS-CoV-2. From this study, it can be concluded that hypo methylation of HMGB1 triggers the expression of this molecule, resulting in the release of HMGB1 into extracellular region. To confirm the role of HMGB1 and SARS-CoV-2 infection with pathogenesis of cancer, further studies are required.

Immune cells have receptors such as toll like receptors (TLR) for HMGB1. Immune cells such as natural killer (NK) cells, CTLs, NKT cells which have cytotoxic functions can recognize extracellularly released HMGB1 from tumor cells $(8,9,19,23,25)$. Elevated levels of HMGB1 have been detected in patients with COAD, DLBC, GBM, LGG, PAAD, READ, STAD and THYM cancer $(17,18)$. We have reported that the HMGB1 expression levels were correlated with the immune infiltration in studied eight cancer types. In humans, it is well known that tumors may behave differently in terms of survival. We did a survival analysis based on the HMGB1 expression of different tumors in relation to immune infiltration through the tumor microenvironment using the Kaplan-Meier plotter. An immune control is responsible for carcinogenesis in human, and is also a research field in terms of providing novel prognostic markers as well as new therapeutic area. In this context, some studies showed the role of HMGB1 expression in various cancer such as gastric, colorectal, pancreatic, esophageal, prostate, bladder, lung cancer and hepatocellular carcinoma $(17,18,26)$. When in silico analysis of intra tumoral immune cells became available in association with HMGB1 expression in studied 8 cancer types, our data show that HMGB1 expression was positively correlated 
a)
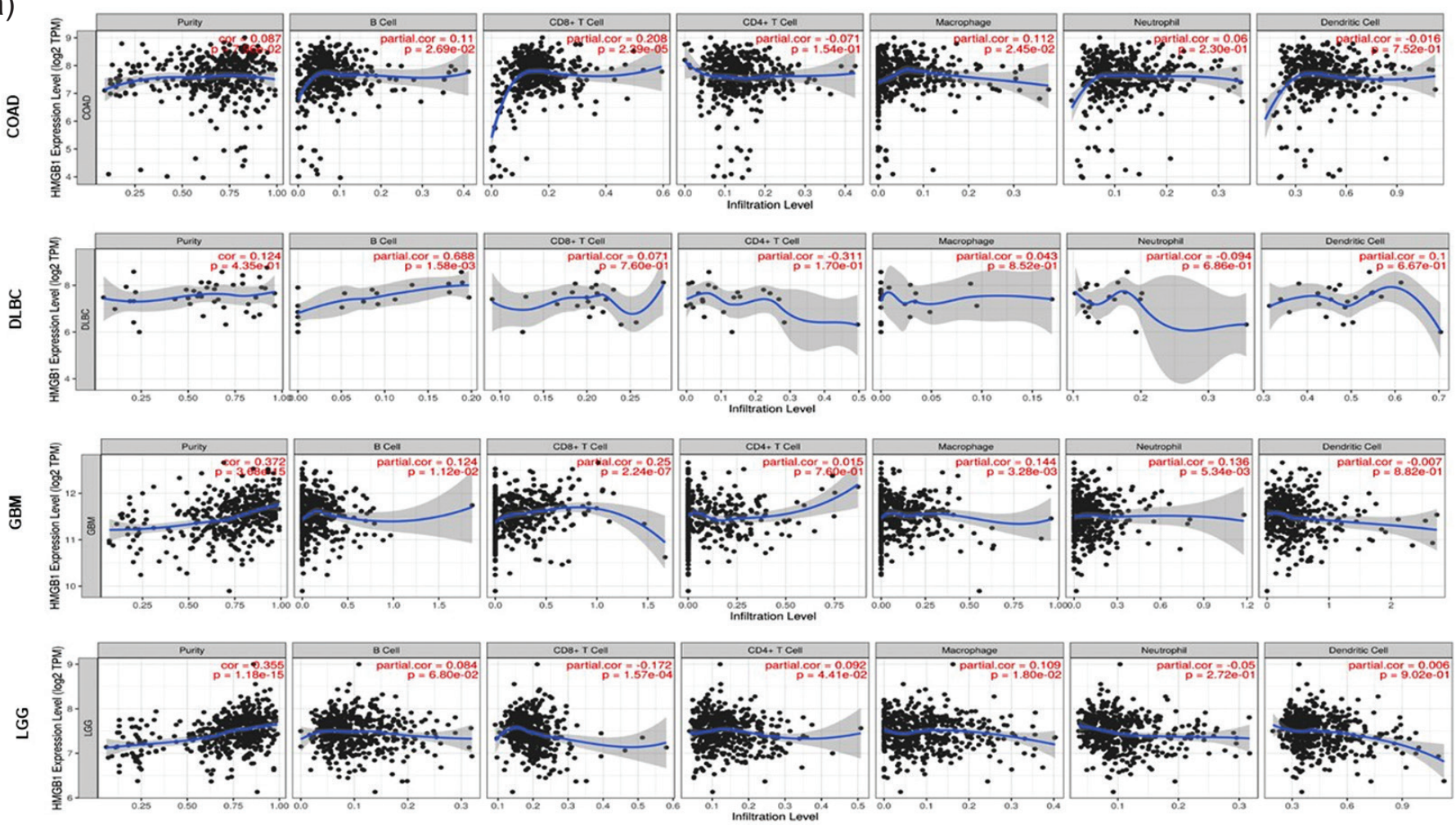

b)
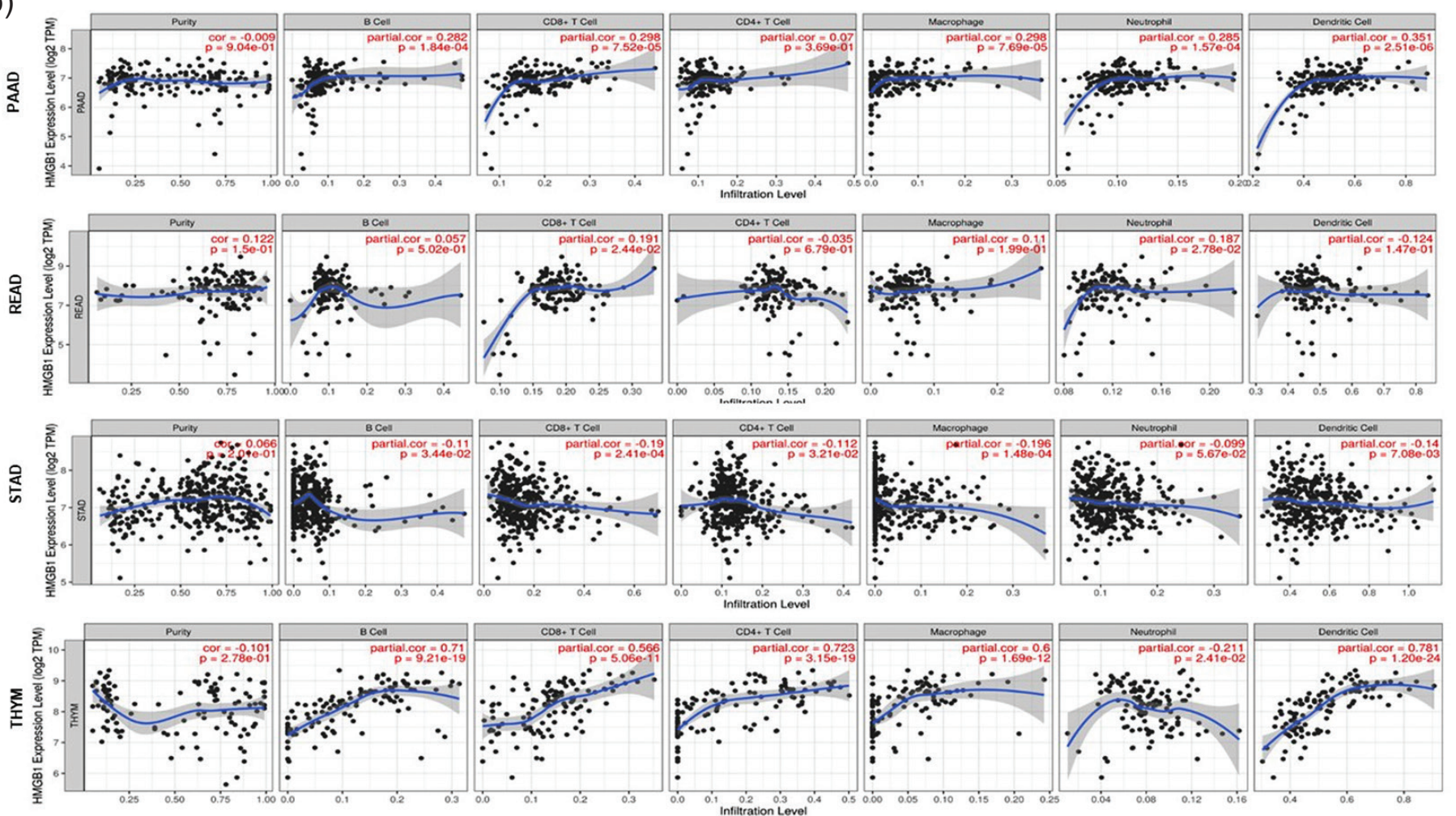

Figure 5. Correlation between HMGB1 expression and immune infiltration in different cancer tissues using TIMER algorithm (a, b)

with B cell, CD8+ T cell, macrophage infiltration in COAD; B cell and DCs in DLBC; B cell, CD8+ T cell, macrophage, neutrophil in GBM; macrophage in LGG; $B$ cell, CD8+ T cell, macrophage, neutrophil, DCs in PAAD; CD8+ T cell, macrophage, neutrophil in READ; B cell, CD8+ T cell, CD4+ T cell, macrophage, DCs in THYM patients tumor samples compared to healthy samples. Recent studies have reported that lymphocytes infiltration in the primary tumor usually correlates with a better clinical outcome in 
cancer patients (27). Our data supported that especially CD8+ cytotoxic $T$ cell infiltration was detected in five tumors of studied eight tumor samples, neutrophil and DCs immune infiltration levels of prostate cancer. Additionally, the level of macrophage in cancer may be related to worst prognosis (28). Our data show that interplay of high expression HMGB1 and CD4+ T helper cell filtrated tumor tissue was very limited or not. The formation of lymphoid structure composing of $\mathrm{T}$ and $\mathrm{B}$ cell was widely detected in cancer patients with highly expressed HMGB1 tissue samples according to our results. Other researchers reported that $\mathrm{T}$ and $\mathrm{B}$ lymphocytes infiltration to tumor tissue was associated with their prognostic value in cancer (29).

Therefore, these results reported herein should be considered in the light of some limitations. One of these limitations is in silico design and lack of experimental study. The other limitation concerns the demographic statistics such as gender and age for healthy group, which could not be downloaded from the database.

\section{Conclusion}

In conclusion, some HMGB1-targeted anti-cancer and antiviral drugs, inhibitors, RNA interference gene silencing are promising in both cancer therapy and also COVID-19 therapy for upregulated HMGB1 cancer types including COAD, DLBC, GBM, LGG, PAAD, READ, STAD and THYM.

\section{Acknowledgements}

The data used in our study are obtained from public database of the TCGA Research Network: https://www.cancer.gov/tcga. We thank the TCGA, GEPIA, TIMER and UALCAN databases for the availability of the data. The datasets generated and analyzed during the current study are available in TCGA database (https:// www.cancer.gov/tcga), the cbio cancer genomics portal (http:// www.cbioportal.org/)

\section{Ethics}

Ethics Committee Approval: Ethics committee approval is not required for the study. Study data were obtained from The Cancer Genome Atlas, and in silico analysis was performed.

Informed Consent: The study is a bioinformatics study, patient consent is not required.

Peer-review: Externally peer-reviewed.

\section{Authorship Contributions}

Concept: R.I.S., Design: R.I.S., Data Collection or Processing: D.F.A-B., Analysis or Interpretation: D.F.A-B., Literature Search: R.I.S., D.F.A-B., Writing: R.I.S., D.F.A-B.

Conflict of Interest: No conflict of interest was declared by the authors.

Financial Disclosure: The authors declared that this study received no financial support.

\section{References}

1. Gonzalez $\mathrm{H}$, Hagerling $\mathrm{C}$, Werb $\mathrm{Z}$. Roles of the immune system in cancer: from tumor initiation to metastatic progression. Genes Dev. 2018;32:1267-1284.

2. Hiraku Y, Guo F, Ma N, et al. Multi-walled carbon nanotube induces nitrative DNA damage in human lung epithelial cells via HMGB1-RAGE interaction and Toll-like receptor 9 activation. Part Fibre Toxicol. 2016;13:16.

3. Ong SP, Lee LM, Leong YF, Ng ML, Chu JJ. Dengue virus infection mediates HMGB1 release from monocytes involving PCAF acetylase complex and induces vascular leakage in endothelial cells. PLoS One. 2012;7:e41932.

4. Wang $H$, Ward MF, Fan XG, Sama AE, Li W. Potential role of high mobility group box 1 in viral infectious diseases. Viral Immunol. 2006;19:3-9.

5. Kempaiah KR, Kurosky A, Hosakote YM. Effects of HMGB1 gene silencing on respiratory syncytial virus-induced inflammatory response. J Immunol. 2018;(Suppl)200:166.

6. Roh JS, Sohn DH. Damage-Associated Molecular Patterns in Inflammatory Diseases. Immune Netw. 2018;18:e27.

7. Kany S, Vollrath JT, Relja B. Cytokines in Inflammatory Disease. Int J Mol Sci. 2019;20:6008.

8. Kang $\mathrm{R}$, Chen $\mathrm{R}$, Zhang $\mathrm{Q}$, et al. HMGB1 in health and disease. Mol Aspects Med. 2014;40:1-116.

9. Nishibori M. [HMGB1 as a representative DAMP and anti-HMGB1 antibody therapy]. Nihon Yakurigaku Zasshi. 2018;151:4-8.

10. Chen Y, Liu Q, Guo D. Emerging coronaviruses: Genome structure, replication, and pathogenesis. J Med Virol. 2020;92:418-423

11. Li H, Liu SM, Yu XH, Tang SL, Tang CK. Coronavirus disease 2019 (COVID-19): current status and future perspectives. Int J Antimicrob Agents. 2020;55:105951.

12. Felsenstein S, Herbert JA, McNamara PS, Hedrich CM. COVID-19: Immunology and treatment options. Clin Immunol. 2020;215:108448.

13. Tufan A, Avanoğlu Güler A, Matucci-Cerinic M. COVID-19, immune system response, hyperinflammation and repurposing antirheumatic drugs. Turk J Med Sci. 2020;50:620-632.

14. Tang Z, Li C, Kang B, Gao G, Li C, Zhang Z. GEPIA: a web server for cancer and normal gene expression profiling and interactive analyses. Nucleic Acids Res. 2017;45:W98-W102.

15. Chandrashekar DS, Bashel B, Balasubramanya SAH, et al. UALCAN: A Portal for Facilitating Tumor Subgroup Gene Expression and Survival Analyses. Neoplasia. 2017;19:649-658.

16. Li T, Fan J, Wang B, et al. TIMER: A Web Server for Comprehensive Analysis of Tumor-Infiltrating Immune Cells. Cancer Res. 2017;77:e108-e110. 
17. Wu T, Zhang W, Yang G, et al. HMGB1 overexpression as a prognostic factor for survival in cancer: a meta-analysis and systematic review. Oncotarget. 2016;7:50417-50427.

18. Zhang L, Han J, Wu H, et al. The association of HMGB1 expression with clinicopathological significance and prognosis in hepatocellular carcinoma: a meta-analysis and literature review. PLoS One. 2014;9:e110626.

19. He SJ, Cheng J, Feng X, Yu Y, Tian L, Huang Q. The dual role and therapeutic potential of high-mobility group box 1 in cancer. Oncotarget. 2017;8:64534-64550.

20. Tripathi A, Shrinet K, Kumar A. HMGB1 protein as a novel target for cancer. Toxicol Rep. 2019;6:253-261.

21. Li G, Liang X, Lotze MT. HMGB1: The Central Cytokine for All Lymphoid Cells. Front Immunol. 2013 Mar 20;4:68.

22. Lü Y, Lu JY, Zhao M, Li ZH, Yang Y. Relationship between HMGB1 content and MHC-II expression in circulating monocytes and spleen of mice challenged with zymosan. Chin J Traumatol. 2009;12:339-343.

23. Andersson U, Ottestad W, Tracey KJ. Extracellular HMGB1: a therapeutic target in severe pulmonary inflammation including COVID-19? Mol Med. 2020;26:42.

24. Street ME. HMGB1: A Possible Crucial Therapeutic Target for COVID-19? Horm Res Paediatr. 2020;93:73-75.
25. Pilzweger C, Holdenrieder S. Circulating HMGB1 and RAGE as Clinical Biomarkers in Malignant and Autoimmune Diseases. Diagnostics (Basel). 2015;5:219-253.

26. Wu L, Yang L. The function and mechanism of HMGB1 in lung cancer and its potential therapeutic implications. Oncol Lett. 2018;15:6799-6805.

27. Fridman WH, Galon J, Dieu-Nosjean MC, et al. Immune infiltration in human cancer: prognostic significance and disease control. Curr Top Microbiol Immunol. 2011;344:124.

28. Poh AR, Ernst M. Targeting Macrophages in Cancer: From Bench to Bedside. Front Oncol. 2018;8:49.

29. Hendry S, Salgado R, Gevaert T, et al. Assessing TumorInfiltrating Lymphocytes in Solid Tumors: A Practical Review for Pathologists and Proposal for a Standardized Method from the International Immuno-Oncology Biomarkers Working Group: Part 2: TILs in Melanoma, Gastrointestinal Tract Carcinomas, Non-Small Cell Lung Carcinoma and Mesothelioma, Endometrial and Ovarian Carcinomas, Squamous Cell Carcinoma of the Head and Neck, Genitourinary Carcinomas, and Primary Brain Tumors. Adv Anat Pathol. 2017;24:311-335. 